\title{
NEUROLOGIC AND MRI ABNORMALITIES IN ACUTE DISSEMINATED ENCEPHALOMYELITIS AND RESPONSE TO PLASMAPHERESIS
}

The relation between the clinical course and MRI findings and response to plasmapheresis were determined by a retrospective record review of 13 children with acute disseminated encephalomyelitis (ADEM) admitted to St Christopher's Hospital for Children, Philadelphia, PA, during 1998-2003. Six of 12 children responded to initial treatment with high-dose methylprednisolone and IV immunoglobulin; one improved spontaneously. The 6 who were unresponsive had a progressive neurologic course, and 5 had delayed onset of MRI abnormalities involving deep gray matter and brainstem. These 5 recovered slowly following plasmapheresis, with residual neurologic deficits. (Khurana DS, Melvin JJ, Kothare SV et al. Acute disseminated encephalomyelitis in children: discordant neurologic and neuroimaging abnormalities and response to plasmapheresis. Pediatries August 2005;116:431-436). (Reprints: Divya S Khurana MD, Section of Neurology, St Christopher's Hospital for Children, Erie Avenue at Front Street, Philadelphia, PA 19134).

COMMENT. MRI abnormalities may appear late in the course of ADEM, leading to a delay in diagnosis and treatment. In almost half the patients in the above series, a significant lag occurred, up to a few weeks, between the presentation of symptoms with fever, ataxia, paresis and mental changes, and the appearance of MRI lesions, usually involving the basal ganglia, thalami, and brainstem, as well as subcortical white matter. Late development of MRI lesions involving deep gray matter and brainstem may be predictive of a prolonged course and lack of response to corticosteroids. The absence of white-matter changes in the MRI up to 3 weeks after onset of symptoms does not exclude a diagnosis of ADEM. Plasmapheresis is recommended in patients with a severe progressive course, unresponsive to corticosteroids and IV immunoglobulin.

\section{BRAIN TRAUMA}

\section{LATE SEQUELAE OF INFLICTED BRAIN INJURY IN INFANTS}

Twenty five children with inflicted traumatic brain injury (TBI) seen in Scotland between 1980 and 1999 were followed prospectively with neurologic and cognitive examinations performed at the Department of Child Life and Health, University of Edinburgh, Scotland. Sixty eight per cent of survivors showed abnormalities at mean followup of 59 months; impairments were severe with complete dependency in $36 \%$, moderate in $16 \%$, and mild in 16\%. Outcome correlated with the Pediatric Trauma and Glasgow Coma Scores but not with age at injury or mechanism of injury. Inflicted TBI has a poor prognosis and correlates with severity of injury. Behavior problems in $52 \%$ began to manifest at age 2 to 3 years, and learning difficulties and attention and memory deficits were recognized only when the child attended school. (Barlow KM, Thomson E, Johnson D, Minns RA. Late neurologic and cognitive sequelae of inflicted traumatic brain injury in infancy. Pediatrics August 2005;116:e174-e185). (Reprints: Dr Karen M Barlow, University of Calgary, Alberta Children's Hospital, 1820 Richmond Rd, Calgary, Alberta, Canada T2T 5C7). 
COMMENT. This study confirms the high morbidity in survivors of TBI in infants, and sequelae may be manifested only after long-term follow-up. The authors emphasize the importance of environmental factors in behavior and development of these patients.

Executive functions after traumatic brain injury in children are reviewed at Baylor College of Medicine, Houston, TX (Levin HS, Hanten G. Pediatr Neurol August 2005;33:79-93). Executive functions include cognitive control (decision making, planning, achieving goals), self-regulation, motivation, and conforming to social behavior. Inhibition is an age-dependent skill linked to cognitive control, and impaired in TBI as well as ADHD. The executive dysfunction following TBI resembles that seen with "developmental" ADHD, and both are related to frontal lobe involvement, which in TBI may be progressive.

\section{SKULL X-RAYS FOR HEAD INJURY}

The effect of abolishing skull X-rays on the rate of admission, use of computer tomography (CT), radiation dose per head injury, and detection of intracranial injuries was determined in patients, aged 1 to 14 years, presenting to the ED at Royal Hospital for Sick Children, Edinburgh, UK. 1535 patients with head injury seen between Aug 1, 1998 and July 31, 1999 (control period) were compared to 1867 presenting between Aug 1, 2002 and July 31,2003 (first year of new skull X ray policy). By abolishing skull X rays, about 400 normal $\mathrm{x}$ rays were avoided, CT orders rose from $1.0 \%$ to $2.1 \%$, with an unchanged positive $\mathrm{CT}$ rate $(25.6 \%$ v $25.0 \%)$, and no change in identification of intracranial injury $(0.2 \%$ v $0.4 \%)$ or in rates of neurosurgical intervention ( $0 \% \vee 0.1 \%)$. The admission rate was unchanged $(10.9 \% \mathrm{v}$ $10.1 \%$ ), and the radiation dose per head injury was slightly decreased from 0.045 to 0.042 $\mathrm{mSv}$. History of the injury and Glasgow coma scale are the best indicators of significant head injury in children. (Reed MJ, Browning JG, Wilkinson AG, Beattie T. Can we abolish skull x rays for head injury? Arch Dis Child August 2005;90:859-864). (Respond: Dr Matthew J Reed, Accident and Emergency Department, Royal Hospital for Sick Children, Sciennes Rd, Edinburgh, UK).

COMMENT. The abolition of skull X-rays in children aged 1 to 14 , presenting with head injury in an ED in the UK, does not increase the admission rate, individual radiation dose, or missed intracranial injury. The mechanism of head injury (falls of $>1$ meter, and road traffic accidents), drowsiness or loss of consciousness, and a low score on the Glasgow coma scale are the most reliable indicators of serious head injury and need for investigation and admission. As pointed out by Tasker RC, in a Commentary (Arch Dis Child 2005;90:774-775), the policy of abolishing skull X-rays doubled the rate of exposure to CT with overall increased risk of radiation. The debate regarding the utility of skull X-rays or the increased use of CT scans in EDs will continue. The above study and that of other guidelines (Dunning J et al. Arch Dis Child 2004;89:763-767) should help in the development of optimal head injury protocols for children. 